\title{
Criminologie
}

\section{Le taux de placement des jeunes en institution : l'écart entre la Belgique et le Québec}

\section{Lucie Lemonde}

Volume 36, numéro 1, printemps 2003

Police et prévention : évaluation et analyse d'impact

URI : https://id.erudit.org/iderudit/006559ar

DOI : https://doi.org/10.7202/006559ar

Aller au sommaire du numéro

\section{Éditeur(s)}

Les Presses de l'Université de Montréal

ISSN

0316-0041 (imprimé)

1492-1367 (numérique)

Découvrir la revue

Citer cet article

Lemonde, L. (2003). Le taux de placement des jeunes en institution : l'écart entre la Belgique et le Québec. Criminologie, 36(1), 177-198.

https://doi.org/10.7202/006559ar
Résumé de l'article

Cet article dresse un portrait comparatif de la situation des mineurs privés de liberté en Belgique francophone et au Québec. Le taux de placement des jeunes en institution publique est beaucoup plus élevé ici qu'en Belgique. Deux facteurs principaux expliquent cet écart important. Premièrement, il est interdit, en Belgique, d'héberger en institution publique les jeunes en danger. Seuls s'y retrouvent des jeunes délinquants. Au Québec, à l'inverse, la très grande majorité des jeunes que l'on retrouve dans les centres de réadaptation sont des jeunes sous protection n’ayant été déclarés coupables d'aucun délit. Une minorité de jeunes contrevenants y sont détenus en vertu d'une condamnation pénale. Deuxièmement, en Belgique, les normes sont presque toutes de nature législative et réglementaire, notamment quant au pouvoir d'envoyer et de maintenir un jeune en institution publique, alors que nos propres normes législatives sont plutôt sommaires et se contentent souvent de déléguer à diverses autorités administratives le pouvoir décisionnel quant au lieu d'hébergement et aux mesures additionnelles de privation de liberté au cours du placement. 


\title{
Le taux de placement des jeunes en institution: l'écart entre la Belgique et le Québec
}

\author{
Lucie Lemonde \\ Professeure \\ Département des sciences juridiques \\ Université du Québec à Montréal \\ lemonde.lucie@uqam.ca
}

RÉSUMÉ - Cet article dresse un portrait comparatif de la situation des mineurs privés de liberté en Belgique francophone et au Québec. Le taux de placement des jeunes en institution publique est beaucoup plus élevé ici qu'en Belgique. Deux facteurs principaux expliquent cet écart important. Premièrement, il est interdit, en Belgique, d'héberger en institution publique les jeunes en danger. Seuls s'y retrouvent des jeunes délinquants. Au Québec, à l'inverse, la très grande majorité des jeunes que l'on retrouve dans les centres de réadaptation sont des jeunes sous protection n'ayant été déclarés coupables d'aucun délit. Une minorité de jeunes contrevenants y sont détenus en vertu d'une condamnation pénale. Deuxièmement, en Belgique, les normes sont presque toutes de nature législative et réglementaire, notamment quant au pouvoir d'envoyer et de maintenir un jeune en institution publique, alors que nos propres normes législatives sont plutôt sommaires et se contentent souvent de déléguer à diverses autorités administratives le pouvoir décisionnel quant au lieu d'hébergement et aux mesures additionnelles de privation de liberté au cours du placement.

ABSTRACT - The article presents a comparison between Quebec and French Belgium's minors deprived of their liberty. The rate of youths sent in public institutions is much higher in Quebec than in Belgium. Two main factors explain this gap. The first one is the fact that in Belgium, it is forbidden to send youths in danger to public institutions, which are only used for young offenders. The situation in Quebec is quite the opposite. The majority of minors that are placed in Quebec rehabilitation centres are youths in need of protection that have not been found guilty of an offence. Only a minority of young offenders are sent to institutions as part of a sentence. The second factor that helps to understand the gap between Belgium and Quebec is the nature of the norms that govern minors and public institutions. In Belgium, the norms are mostly laws and regulations, and it is notably the case with the power to send and maintain youths in public institutions. In Quebec, most norms are summary rules and the power to decide about the custody/ placement of youths - as of other liberty deprivation measuresare often delegated to diverse administrative authorities. 
Cet article dresse un portrait comparatif de la situation des mineurs privés de liberté en Belgique francophone et au Q uébec ${ }^{1}$. II n'existe en Belgique francophone que 240 places en internat dans les institutions publiques de protection de la jeunesse (IPPJ) pour une population d'environ quatre millions et demi d'habitants. De ce nombre, on retrouve 50 places en régime fermé et 190 places en régime ouvert. Les autres enfants en danger sont hébergés à l'externe dans des résidences privées agréées (genre foyers de groupe). Au Q uébec par contre, on retrouve, dans les seuls centres de réadaptation de M ontréal, près de 600 places en internat et, selon les chiffres du ministère de la Santé et des Services sociaux², il existe, dans l'ensemble de la province, 3050 places en internat ${ }^{3}$. D e ces 3050 places, aucune donnée provinciale globale ne spécifie le nombre de places en régime ouvert ou en régime fermé.

Comment expliquer cet écart colossal entre les deux communautés? Comment expliquer le fait qu'au Q uébec nous enfermions dix fois ${ }^{4}$ plus de jeunes? Parmi les nombreuses différences juridiques et sociales ${ }^{5}$, deux facteurs sont déterminants. Premièrement, il est interdit, en Belgique, $d$ 'héberger en institution publique les jeunes en danger. Seuls s'y retrouvent des jeunes délinquants ayant commis, selon l'expression consacrée, des faits qualifiés d'infraction. Au Q uébec, à l'inverse, trois lois distinctes peuvent conduire un jeune dans un centre de réadaptation: la Loi sur la protection de la jeunesse (LPJ), la L oi sur les services de santé ou de services sociaux

1. Grâce à des subventions du FCAR et du CQRS accordées pour une recherche portant sur les droits des mineurs privés de liberté, j'ai pu visiter les institutions publiques de protection de la jeunesse en Belgique et m'entretenir avec plusieurs personnes des milieux institutionnel, associatif et universitaire. J'ai également fait de l'observation participante dans trois unités de vie des $C$ entres jeunesse de M ontréal et ai eu des entretiens avec des responsables, des éducateurs et des jeunes.

2. Voir <http:/ / www.msss.gouv.qc.ca/ f/ reseau/ etabliss.htm>.

3. Les centres de réadaptation du Q uébec comprennent des unités ouvertes et fermées dans des établissements publics, ainsi que des services résidentiels dans des foyers de groupe publics ou privés. Cet article ne s'intéresse qu'à l'hébergement des mineurs en internat et non dans les foyers de groupe.

4. Les taux de places à l'interne ont été calculés sur une base de 100000 habitants. En Belgique francophone, le taux est de 4,6 places pour les mineurs privés de liberté pour 100000 habitants, alors que ce taux est de 42,2 au Q uébec.

5. Deux auteurs, I'un québécois, Jean Trépanier, et l'autre belge, Françoise Tulkens, ont étudié les deux systèmes de justice pour mineurs dans une perspective historique. Comme ils l'écrivent: "Autant dans le passé qu'actuellement, l'évolution du droit des mineurs est inséparable de la politique économique et sociale, de la politique criminelle, de la politique culturelle... (Trépanier et Tulkens, 1995 : 13). 
(LSSSS) et la L oi sur les jeunes contrevenants (LJC $)^{6}$. Les deux premières relèvent du législateur provincial et elles concernent la réadaptation des enfants ayant besoin de protection ou présentant des troubles de comportement sérieux. La dernière relève du législateur fédéral et vise la responsabilisation des jeunes ayant commis une infraction criminelle et la protection de la société. La très grande majorité des jeunes que l'on retrouve dans les centres de réadaptation sont des jeunes sous protection n'ayant été déclarés coupables d'aucun délit. Une minorité de jeunes contrevenants y sont détenus en vertu d'une condamnation pénal $e^{7}$. D euxièmement, en Belgique, les normes sont presque toutes de nature législative et réglementaire, notamment quant au pouvoir d'envoyer et de maintenir un jeune en institution publique, alors que nos propres normes législatives sont plutôt sommaires et se contentent souvent de déléguer à diverses autorités administratives le pouvoir décisionnel quant au lieu d'hébergement et aux mesures additionnelles de privation de liberté au cours du placement.

N ous nous attarderons tout d'abord sur les sources et le contenu des normes applicables, pour soulever ensuite quelques éléments de discussion expliquant cet écart.

\section{A. Les normes législatives et réglementaires relatives au placement en institution}

Dans les deux pays, le placement initial est régi par la loi et est le fruit d'une ordonnance judiciaire ${ }^{8}$. L'imposition d'une mesure d'hébergement à l'endroit des jeunes délinquants est de compétence fédérale dans les

6. Le gouvernement fédéral a adopté une nouvelle loi, la L oi sur le système de justice pénale pour les adolescents, C-7, 2002, remplaçant la LJC, qui entrera en vigueur en avril 2003. Dans ce texte, nous parlerons de la LJC et soulignerons, lorsque nécessaire, les modifications pertinentes de la nouvelle loi. Cette nouvelle loi est beaucoup plus répressive et soumet plusieurs catégories d'adolescents au régime adulte.

7. II n'existe pas de statistiques provinciales à jour sur la «clientèle» des centres de réadaptation; il faut donc s'en tenir au recensement effectué par Camille M essier et Jean Toupin en 1991. Selon ces chercheurs, la très grande majorité des adolescents (75\%) - la majorité des garçons (62\%) et presque toutes les filles $(93 \%)$ - sont des jeunes en protection, alors que seulement un sur cinq (21\%) est un jeune contrevenant et, dans ce dernier cas, il s'agit presque uniquement de garçons (M essier et Toupin, 1994 : 107).

8. II existe une exception à cette règle générale au Q uébec. Un jeune peut être admis dans un centre sur une base volontaire en vertu de la LPJ et de la LSSSS. II y a lieu de se poser des questions sur le caractère libre et volontaire d'un telle admission. Dans le cadre de la LPJ, ni les parents, ni l'enfant n'ont sollicité le placement. C'est le directeur de la protection de la jeunesse qui le propose, et si les parents ou l'enfant refusent, le dossier est automatiquement judiciarisé (LPJ, art. 52 ; C entre de recherche sur les droits et libertés, 1985). 
deux pays ${ }^{9}$, mais les deux législations confient à l'autorité provinciale ou communautaire l'application d'une telle mesure ${ }^{10}$. S'agissant des jeunes en danger ou sous protection, le placement est de compétence provinciale ou communautaire ${ }^{11}$.

\section{L'ordonnance de placement des jeunes délinquants}

En droit belge, les jeunes de moins de 18 ans $^{12}$ ne peuvent pas commettre d'infraction et ne tombent donc pas sous le coup du droit pénal classique en vertu d'une présomption irréfragable de non-discernement ${ }^{13}$. Avant cet âge, les jeunes ayant commis un fait qualifié d'infraction sont soumis à des mesures de garde, de préservation ou d'éducation qui peuvent comprendre un hébergement en IPPJ pour une durée déterminée, en régime ouvert ou fermé. C ette décision est judiciaire, tant en ce qui a trait à sa durée qu'au type de régime, ouvert ou fermé.

En effet, selon le paragraphe 4 de l'article 37 de la Loi du 8 avril 1965 relative à la protection de la jeunesse, le tribunal peut confier les jeunes ayant commis un fait qualifié d'infraction à une institution publique d'observation et d'éducation. La décision doit préciser la durée de la mesure et spécifier si elle doit se dérouler en régime ouvert ou fermé. La mesure de mise sous garde en régime fermé ne peut être prise qu'en cas de mauvaise conduite persistante ou de comportement dangereux du jeune et elle ne

9. Soit la LJC au Canada et la Loi du 8 avril 1965 relative à la protection de la jeunesse en Belgique.

10. Voir art. 24 de la LJC, au Canada. En Belgique, I'article 5\#1 Loi du 8 août 1980 des réformes institutionnelles, s'appuyant sur l'article 128 de la Constitution, confie la compétence sur "la protection de la jeunesse, en ce compris la protection sociale et la protection judiciaire», aux Communautés française et flamande, à l'exception toutefois « de la détermination des mesures qui peuvent être prises à l'égard des mineurs ayant commis un fait qualifié d'infraction ». C'est donc un décret communautaire qui régit ce domaine, plus particulièrement le D écret du 4 mars 1991 de la Communautéfrançaise relatif à l'aide à la jeunesse.

11. Soit la LPJ au Q uébec et le D écret du 4 mars 1991 de la Communauté française relatif à l'aide à la jeunesse en Belgique.

12. L'âge de la responsabilité pénale est fixé à 18 ans en Belgique, alors qu'il est de 12 ans au Canada.

13. II existe deux exceptions à cette règle générale. Un jeune entre 16 et 18 ans soupçonné d'infractions relatives ou connexes au « roulage» (entendre la conduite automobile) peut être déféré aux juridictions de droit commun (art. 36bis de la L oi du 8 avril 1965 relativeà la protection de la jeunesse en Belgique). De la même façon, le tribunal de la jeunesse peut se dessaisir du dossier d'un jeune entre 16 et 18 ans soupçonné d'un fait qualifié d'infraction et renvoyer I'affaire au ministère public aux fins de poursuites devant les tribunaux de droit commun (art. 38 de cette même Loi). 
doit pas être appliquée aux jeunes de moins de 12 ans, "sauf circonstances très exceptionnelles». II faut dire que le régime fermé, dans les IPPJ belges, ressemblent fort à la prison: la liberté de mouvement des jeunes est sévèrement contrôlée de même que leurs droits de sortie; l'aménagement des lieux est typique d'une aile de prison, les portes des cellules sont pleines avec un petit vasistas, l'ameublement est rudimentaire, les portes se verrouillent et ainsi de suite.

Le placement en milieu fermé est d'une durée maximale de trois mois, renouvelable une seule fois après réception d'un rapport médico-psychologique et après que l'intéressé et son conseil ont été entendus. II peut néanmoins être prolongé de mois en mois par décision judiciaire motivée et justifiée par « des circonstances graves et exceptionnelles se rattachant aux exigences de la sécurité publique ou propres à la personnalité de l'intéressé, et qui nécessitent le maintien de ces mesures ${ }^{14} »$.

II faut mentionner ici une particularité du droit belge. En vertu de l'article 53 de la L oi du 8 avril 1965, lorsqu'il était matériellement impossible de trouver une place sur-le-champ dans une IPPJ pour un mineur soupçonné d'avoir commis un fait punissable d'une peine d'emprisonnement correctionnelle d'un an ou plus, celui-ci pouvait être gardé provisoirement dans une maison d'arrêt pour une période maximale de quinze jours. Cette phrase est au passé car cet article a été abrogé le $1^{\text {er }}$ janvier 2002. La ministre de l'Aide à la jeunesse a annoncé les mesures prises pour que les IPPJ puissent accueillir ces jeunes. Le nombre de places en régime fermé a été augmenté de 38 à 50 places et il existe de nouvelles mesures alternatives au placement en IPP ${ }^{15}$. Selon le directeur adjoint de I'IPPJ de Braine-le-Château, les séjours seront plus courts et il y aura ainsi un plus grand roulement. De plus, un programme dit d'accompagnement postrésidentiel intensif a été mis sur pied pour ces jeunes en fin de séjour.

14. Article 52 quater, de la Loi du 8 avril 1965, modifié par la Loi du 2 février 1994. Selon un entretien que j'ai eu avec le directeur de I'IPPJ de Fraipont, M. Demoulin, la durée moyenne d'un placement en régime fermé est de quatre mois et demi.

15. Question no 34 du 19 juillet 2000 de M. Diaf au ministre de l'Aide à la jeunesse, rapportée dans le Journal du droit des jeunes, no 205, mai 2001, p. 32. Depuis que ces lignes ont été écrites, un A ccord de coopération entre l'É tat fédéral, la Communauté germanophone, la Communauté française et la Communauté flamande rel atif au centre fermé pour le placement provisoire de mineurs ayant commis un fait qualifié d'infraction a été passé. Cet accord prévoit la création, à EverbergKortenberg, d'un centre fermé pour garçons d'une capacité maximale de 50 places (24 pour les francophones, 24 pour les néerlandophones et 2 pour les germanophones). 
Au Q uébec, le placement des jeunes délinquants en centre de réadaptation a été, jusqu'à maintenant, solidement encadré par la loi et relevait entièrement du pouvoir judiciaire. En vertu de la LJC, seul le tribunal pouvait ordonner la mise sous garde pour une période déterminée en centre de réadaptation (LJC, art. 20). II devait spécifier dans son ordonnance le type de garde imposée, soit en milieu ouvert ou en milieu fermé (LJC, art. 24.1(2)). D ans la nouvelle L oi sur le système de justice pénale pour les adolescents, le choix du lieu de garde ne relève plus du pouvoir judiciaire, mais il est confié au directeur provincial, soit au Q uébec le directeur de la protection de la jeunesse. L'article 85 (1) spécifie que, dans chaque province, le régime de garde et de surveillance applicable aux adolescents doit offrir au moins deux niveaux de garde qui se distinguent par le degré de confinement. Le paragraphe (3) de ce même article prévoit que lorsqu'une ordonnance de placement est prononcée, c'est le directeur provincial qui détermine le niveau de garde indiqué pour le placement de l'adolescent après avoir pris en compte les facteurs suivants: le niveau de garde imposé doit être le moins élevé possible, compte tenu de la gravité de l'infraction et des circonstances de sa perpétration, des besoins de l'adolescent et de sa situation personnelle- notamment la proximité de la famille, d'une école, d'un emploi et de services de soutien - de la sécurité des autres adolescents sous garde et de l'intérêt de la société; le niveau de garde imposé doit permettre la meilleure adéquation possible entre le programme destiné à l'adolescent, d'une part, et les besoins et la conduite de celui-ci, d'autre part, compte tenu des résultats de son évaluation et des risques d'évasion.

La détermination du niveau de garde, soit plus spécifiquement le placement en régime fermé des jeunes délinquants, relève donc du pouvoir judiciaire en Belgique, comme c'était le cas au Canada jusqu'à maintenant. Cette responsabilité relèvera désormais de l'autorité administrative.

\section{L'ordonnance de placement des jeunes en danger}

En Belgique, comme on l'a vu, un jeune en danger qui n'a commis aucun délit ne peut être hébergé dans une institution publique. L'article 37 de la Loi du 8 avril 1965 spécifie que le tribunal de la jeunesse peut ordonner des mesures de garde, de préservation ou d'éducation à l'endroit des jeunes en danger. Plus spécifiquement, il peut les réprimander, les soumettre à la surveillance du service social compétent tout en les maintenant dans leur milieu, les placer chez une personne digne de confiance 
ou dans un établissement approprié en vue de leur hébergement, de leur traitement, de leur éducation, de leur instruction ou de leur formation professionnelle.

Le D écret du 4 mars 1991 de la Communauté française relatif à l'aide à la jeunesse s'applique aux jeunes en difficulté quels qu'ils soient, mais plus particulièrement aux enfants dont la santé ou la sécurité est en danger ou dont les conditions d'éducation sont compromises par son comportement, celui de sa famille ou de ses familiers. II régit également l'application des mesures judiciaires à l'endroit de jeunes qui ont commis des faits qualifiés d'infractions. La détermination de telles mesures est, rappelons-le, de compétence fédérale, mais leur application est de compétence communautaire. L'article 3 du décret stipule que tout enfant visé a droit à une aide spécialisée qui tend «à lui permettre de se développer dans des conditions d'égalité des chances en vue de son accession à une vie conforme à la dignité humaine».

Selon ce décret, un placement dans une institution publique est réservé aux jeunes délinquants ayant commis un fait qualifié d'infraction et relève exclusivement du pouvoir judiciaire et non du conseiller d'aide à la jeunesse, car il s'agit d'une «aide imposée» (D écret, art. 16). Ceci se justifie, selon l'exposé des motifs, par le fait que «le pouvoir judiciaire demeure le meilleur garant du respect des droits».

Les jeunes en danger qui n'ont pas commis un fait qualifié d'infraction ne peuvent en aucun cas être placés dans une IPPJ ${ }^{16}$. Ils sont dirigés vers d'autres lieux: familles d'accueil, résidence privée ouverte ayant été agréée. Ils ne peuvent jamais, a fortiori, se retrouver en régime fermé. Selon l'article 18 du décret, I'hébergement en régime fermé ne peut être confié qu'à un établissement faisant partie des IPPJ. A contrario, les services résidentiels privés ne peuvent donc pas mettre sur pied des régimes fermés. Selon Tulkens et M oreau, il s'agit là « de la mise en œuvre d'un des principes directeurs du décret: I'exécution d'une mesure privative de liberté est un monopole exclusif de la puissance publique» (2001: 383).

Au Q uébec, en ce qui concerne les jeunes en besoin de protection, l'article 91 de la LPJ prévoit que, si le tribunal en vient à la conclusion que la sécurité ou le développement d'un enfant est compromis, il peut ordonner diverses mesures. Celles-ci vont du maintien en milieu familial avec surveillance au placement dans «un établissement qui exploite un

16. II semble toutefois que l'on cherche un «délit-prétexte» pour pouvoir enfermer certains jeunes (Graindorge, 2001). 
centre de réadaptation ou à une famille d'accueil, choisi par l'établissement». L'article 62 de la LPJ précise que, lorsque le tribunal ordonne I'hébergement obligatoire d'un enfant, il charge le directeur de la protection de la jeunesse de désigner l'établissement auquel l'enfant sera confié.

En d'autres mots, ce n'est pas le tribunal qui décide si un jeune en protection sera hébergé en régime fermé ou ouvert. II existe toute une controverse jurisprudentielle sur le pouvoir de désignation exacte du lieu $d$ 'hébergement des jeunes sous protection. C ertains juges se considèrent en droit de désigner l'unité qui devra accueillir le jeune en s'appuyant sur l'article 91 al. 2, d) de la LPJ qui énonce que le juge peut «faire toute autre recommandation dans l'intérêt de l'enfant». D'autres, à l'inverse, refusent de le faire en arguant que le pouvoir de désignation d'un centre ou d'une unité est exclusivement réservé au directeur de la protection de la jeunesse, lequel connaît mieux les ressources existantes. D ésigner serait une fonction administrative plutôt que judiciaire. D 'autres enfin adoptent une position mitoyenne. Si le tribunal ne peut désigner nommément un centre, il peut toutefois préciser le degré sécuritaire requis ou exclure certaines unités jugées inappropriées aux besoins du jeune et au respect de ses droits (Boulais, 1999; Lemonde, 2001).

Selon la dernière décision de la Cour d'appel du Q uébec sur la question, le tribunal décide de l'hébergement obligatoire d'un enfant et la direction de la protection de la jeunesse désigne le centre de réadaptation ou la famille d'accueil qui prendra charge de l'enfant (M .V . C. $G$ agnon, 2002). Q uoi qu'il en soit, la quasi-totalité, sinon la totalité, des décisions en ce qui a trait au choix du lieu d'hébergement échappe au pouvoir judiciaire.

Chaque centre de réadaptation se divise en différentes unités de réadaptation, communément appelées «unités de vie». Ces différentes unités ne s'équivalent pas toutes. Bien que la réal ité de chaque centre de réadaptation varie suivant les régions, on y retrouve toujours deux types d'unités, soit les unités régulières ou gl obalisantes, qui accueillent les jeunes sous protection de même que les jeunes contrevenants condamnés à une mesure de mise sous garde en régime ouvert suivant la LJC, et les unités de garde fermée, qui sont des unités sécuritaires accueillant exclusivement les jeunes contrevenants condamnés à une mesure de garde fermée suivant la LJC. Un troisième type d'unité a été créé dans les centres jeunesse, soit les unités d'encadrement intensif que l'on qualifie d'unités ouvertes, mais dont les conditions de vie et les aménagements architecturaux sécuritaires ressemblent tout à fait à ce qui existe en garde fermée. $0 \mathrm{n}$ y 
retrouve des jeunes contrevenants condamnés à une mise sous garde en milieu ouvert et des jeunes sous protection.

Le degré de liberté varie énormément entre ces types d'unités. D ans les unités sécuritaires, soit en garde fermée et en encadrement intensif, la porte de l'unité est toujours fermée à clef, la porte de la chambre du jeune se barre automatiquement sitôt fermée, la cour attenante à l'unité est entourée de hautes clôtures, les fenêtres peuvent être grillagées, les déplacements des jeunes sont strictement contrôlés, et ainsi de suite.

$C$ 'est donc dire que, contrairement à la situation qui prévaut en Belgique, non seulement les jeunes $Q$ uébécois en danger qui n'ont pas été déclarés coupables de délits peuvent être placés en institution publique, mais ils peuvent de surcroît se retrouver en régime fermé et ainsi passer des mois, voire des années, dans une unité d'encadrement intensif.

\section{B. Les normes législatives et réglementaires relatives aux transferts}

II peut arriver qu'au cours de son placement un jeune soit transféré d'une institution à une autre ou d'un régime ouvert à un régime sécuritaire. Ces transferts entraînent souvent une privation additionnelle de liberté.

En Belgique francophone, les transferts disciplinaires sont interdits. Les transferts sont régis par la loi. Selon l'article 15 du D écret du 4 mars 1991 de la Communauté française relatif à l'aide à la jeunesse, toute décision de transfert d'un lieu résidentiel à un autre doit être prise par l'autorité qui a ordonné le placement. Sauf pour des raisons médicales ou de sécurité, aucun transfert ne peut être effectué avant d'avoir obtenu l'accord du jeune concerné, s'il a plus de 14 ans, ou des personnes qui en assument la garde, s'il n'a pas atteint cet âge. Selon l'exposé des motifs, «la ratio legis de cet article est de mettre fin à la pratique illégale des transferts disciplinaires déguisés. [...] D ans tous les cas, le jeune et les membres de sa famille peuvent contester une décision de transfert à laquelle ils n'auraient pas été associés et soumettre la contestation à l'arbitrage du tribunal de la jeunesse» (Exposé des motifs, 1990: 17).

$\mathrm{Au} \mathrm{C}$ anada, en ce qui concerne les jeunes contrevenants, la LJC exigeait que ce soit le tribunal qui, dans son ordonnance, décide du type de garde, ouverte ou fermée. Le directeur pouvait transférer l'adolescent d'une garde ouverte à une garde fermée, mais pour une période de 15 jours seulement et uniquement si celui-ci s'évadait ou tentait de le faire, ou si c'était nécessaire pour la sécurité d'une personne ou de l'établisse- 
ment. Le jeune contrevenant pouvait s'adresser au tribunal pour un examen de ce transfert en vertu de l'article 28.1 de cette même loi. Comme on l'a vu, la nouvelle Loi sur le système de justice pénale pour les adolescents n'exige plus que ce soit le tribunal qui décide du type de garde des jeunes contrevenants. C'est le directeur de la protection de la jeunesse qui en est chargé. Ce dernier peut donc tout aussi bien, et suivant les mêmes critères, décider de transférer un adolescent d'une garde ouverte à une garde fermée. L'article 85 (4) le prévoit d'ailleurs sans ambiguïté: «Le directeur provincial peut, après avoir pris en compte les facteurs prévus au paragraphe (5), décider de faire passer l'adolescent d'un niveau de garde à un autre, s'il est convaincu que cette mesure est préférable dans l'intérêt de la société et eu égard aux besoins de l'adolescent. »

Au Q uébec, les transferts des jeunes sous protection sont fréquents et ne sont pas encadrés par la loi. La loi est muette sur le pouvoir de transférer les jeunes d'un lieu à l'autre. II n'existe aucun classement législatif ou réglementaire des diverses unités en fonction de leur degré de sécurité. La décision de maintenir un jeune dans une unité ouverte ou fermée, de le transférer d'un régime à l'autre, n'est pas le fruit d'une ordonnance judiciaire, même si elle comporte souvent une privation additionnelle de liberté.

Avant 1984, la LPJ contenait une définition d'unité sécuritaire. L'ancien article $1 \mathrm{~h}$ ) énonçait en effet qu'une unité sécuritaire était:

un endroit, caractérisé par un aménagement architectural plus limitatif, situé dans un centre d'accueil, où sont dispensés des services de réadaptation visant la réintégration sociale de l'enfant et où sont appliquées des règles internes particulières visant à contrôler les déplacements de l'enfant en vue de lui venir en aide, tout en protégeant la société.

Le pouvoir d'envoyer une jeune personne dans un milieu sécuritaire appartenait alors au tribunal de la jeunesse en vertu de l'article 91e) de la LPJ qui prévoyait que celui-ci pouvait ordonner «l'hébergement obligatoire d'un enfant âgé de 14 ans ou plus dans une unité sécuritaire, pour une période maximale de trois mois, s'il est d'avis que l'enfant tentera de se soustraire à l'application de la loi ou qu'il représente un danger pour lui-même ou pour autrui ».

L'hébergement dans une unité sécuritaire était une mesure distincte qui ne pouvait pas être ordonnée par le directeur de la Protection de la jeunesse, lequel ne pouvait non plus modifier une ordonnance du tribunal (PJ-193, 1986). L'ancien article 46 permettait toutefois au directeur de faire héberger provisoirement un enfant en milieu sécuritaire, en cas d'urgence, mais il ne pouvait pas le faire pour plus de 24 heures sans 
autorisation du tribunal. Les mêmes motifs de danger ou de risque de fugue étaient ici également requis.

Aujourd 'hui, non seulement la loi actuelle est-elle silencieuse quant à la durée et aux motifs pouvant justifier le placement ou le transfèrement d'un enfant en milieu sécuritaire, mais elle ne prévoit pas non plus de mécanisme judiciaire de contrôle préalable de l'utilisation des unités sécuritaires. Tout ce que la LPJ prévoit, c'est que l'enfant et ses parents doivent être informés et consultés avant un transfert dans un autre établissement. Cet article 7 ne s'appliquerait donc pas aux transferts intraétablissements. D e plus, la jurisprudence est à l'effet que consulter quelqu'un ne signifie pas débattre de la question avec lui et n'impose pas qu'on doive attendre une adhésion ou un consentement avant d'y procéder (Boulais, 1999: 63).

\section{Les normes et les pratiques relatives à l'isolement et aux mesures disciplinaires}

Tous les centres de réadaptation ou les institutions publiques ont des salles d'isolement où peuvent être enfermés des jeunes pour des périodes de temps plus ou moins longues. Les salles d'isolement sont, à première vue, assez semblables dans les institutions belges et les centres québécois. Ce sont de petites pièces nues, entièrement vides ou ne comprenant qu'un lit sans drap. Une fenêtre à même la porte verrouillée permet de surveiller l'enfant qui y est enfermé.

En Belgique, cependant, l'article 19.5 du D écret du 4 mars 1991 de la Communauté française relatif à l'aide à la jeunesse édicte que l'exécutif doit réglementer les modalités de l'isolement et fixer des normes respectueuses de la dignité humaine. Des normes applicables aux locaux d'isolement ont effectivement été fixées dans un A rrêté du G ouvernement de la Communauté française régl ementant les modalités d'isolement. II est ainsi prévu que tout local servant de lieu d'isolement doit avoir les dimensions minimales de $9 \mathrm{~m}^{2}$ de surface et $22 \mathrm{~m}^{3}$ de volume, doit être éclairé naturellement, donc avoir une fenêtre laissant passer la lumière du jour ${ }^{17}$. Il doit comprendre au minimum un lit ou un bas-flanc, une table et un siège, de même qu'il

17. Les salles d'isolement que j'ai visitées ont effectivement de larges fenêtres. Cependant, à I'IPPJ de Braine-le-Château, les fenêtres sont munies d'un store électrique en fer que seul l'intervenant peut ouvrir. 
doit permettre l'accès à des installations sanitaires garantissant I'hygiène. Dans tous les cas, il doit être convenablement entretenu, chauffé et ventilé et le jeune doit recevoir une tenue vestimentaire décente et, au moment du coucher, un pyjama.

Au Q uébec, les lieux d'isolement ne sont régis par aucune norme législative ou réglementaire. Les salles d'isolement que nous avons visitées sont beaucoup plus petites que celles vues en Belgique, elles n'ont pas de fenêtre donnant sur l'extérieur ni table ou siège, ni installation sanitaire dans la pièce même.

Le cadre législatif entourant la décision d'envoyer un enfant en isolement est également beaucoup plus strict en Belgique. L'article 19 du D écret du 4 mars 1991 de la Communauté française relatif à l'aide à la jeunesse prévoit qu'une mesure d'isolement ne peut être prise qu'à l'intérieur d'une IPPJ (donc jamais à l'égard d'un jeune en danger) et qu'à l'endroit d'un jeune qui compromet sa sécurité physique ou celle des autres jeunes, du personnel ou des visiteurs. Toute mesure d'isolement doit être communiquée sur-le-champ à l'autorité de placement et elle ne peut être prolongée au-delà de 24 heures sans l'accord du juge compétent. Celui-ci doit rendre une décision motivée, dans le respect des droits de l'intéressé, et autoriser la prolongation de l'isolement pour un délai qu'il précise et qui ne doit pas dépasser huit jours. La mesure doit être levée dès que cesse la situation qui la motive. Le Règlement général du groupe des institutions publiques de protection de la jeunesse de la communauté française ajoute quant à lui que le jeune placé en isolement doit recevoir des visites quotidiennes de l'équipe de direction, de l'équipe scientifique et médicale ainsi que celle, toutes les deux heures, de l'équipe éducative.

La possibilité d'isoler un enfant pour une période de huit jours apparaît excessive d'autant plus qu'elle peut être renouvelée une fois. Plusieurs recherches ont démontré que l'isolement doit être de très courte durée et que prolonger la période d'isolement est inutile et contre-indiqué (Bandeira et 0 uellet, 1986 ; C respi, 1988 ; Kennedy et al., 1990 ; M ace et Heller., 1990).

Au Q uébec, l'article 118.1 LSSSS stipule que:

La force, l'isolement, tout moyen mécanique ou toute substance chimique ne peuvent être utilisés comme mesure de contrôle d'une personne dans une installation maintenue par un établissement, que pour l'empêcher de s'infliger ou d'infliger à autrui des lésions.

L'utilisation d'une telle mesure doit être minimale et exceptionnelle et doit tenir compte de l'état physique et mental de la personne. 
Il est également prévu que l'utilisation de la mesure d'isolement doit être consignée de façon détaillée dans le dossier personnel en décrivant les moyens utilisés, la période et le comportement qui a motivé la mesure.

L'Association des Centres jeunesse s'est dotée d'un cadre de référence pour I'utilisation de l'isolement (ACJ, 1995) et chaque centre jeunesse a adopté un règlement interne sur le sujet. Pour l'Association des centres jeunesse, l'isolement est une mesure exceptionnelle à l'endroit d'un jeune qui doit durer «juste le temps nécessaire d'arrêter sa perte de contrôle dangereuse» (AC), 1995, art. 6). Les situations de perte de contrôle dangereuse sont énumérées à l'article 7, soit l'agression physique, l'automutilation, la destruction de l'environnement, I'agression verbale et le comportement désorganisé. Cette liste embrasse beaucoup plus de situations que la loi ne le permet puisque celle-ci ne mentionne que le fait de "s'infliger ou d'infliger à autrui des lésions». Le règlement des Centres jeunesse de M ontréal ne reprend pas cette liste (CJM , 1995). L'article 3 parle également de "perte de contrôle dangereuse», qu'il définit comme étant un état de désorganisation au cours duquel le jeune est incapable d'un jugement adéquat sur les conséquences de ses actes, et lorsque le jeune se comporte de façon constituant un danger réel ou imminent pour lui-même ou les autres.

À première vue, il semble que la mesure d'isolement soit solidement encadrée dans les deux pays quoique cet encadrement soit plus serré en Belgique puisque le corpus normatif est législatif plutôt qu'institutionnel, que la loi précise que la mesure d'isolement ne peut excéder 24 heures sans l'accord d'un juge, alors que les règles proposées par l'Association des $C$ entres jeunesse et le règlement des $C$ entres jeunesse de M ontréal ne précisent aucun délai, mais parlent « du temps nécessaire pour arrêter la perte de contrôle» et, finalement, puisque les normes belges s'attachent également à l'aménagement physique des lieux.

De plus, si l'isolement est régi par l'article 188.1 LSSSS, il semble bien que le mot «isolement» ait une portée très étroite dans les centres de réadaptation. Seule une mesure d'isolement se déroulant dans une salle d'isolement serait considérée comme telle; une mesure disciplinaire se déroulant dans une salle de retrait, fût-elle exactement semblable à la salle d'isolement, i.e. complètement vide et fermée à clef, ne constituerait pas de l'isolement et échapperait aux exigences de la LSSSS.

Le retrait est une intervention disciplinaire qui consiste à retirer un jeune du groupe. Le retrait se déroule habituellement dans la chambre du jeune. Dans les unités d'encadrement intensif, la mesure de retrait en 
chambre s'effectue porte verrouillée. II existe également des chambres de retrait, soit des pièces exclusivement désignées à cette fin. Celles que nous avons vues (aux centres de réadaptation Dominique-Savio-M ainbourg et Cité-des-Prairies) s'apparentent aux salles d'isolement: pièce vide, sans fenêtre, porte verrouillée. Plusieurs chambres de retrait sont parfois regroupées en une seule unité, appelée «bloc-retrait». C'est le cas de l'unité La R elance, au centre Cité-des-Prairies.

La mesure de retrait est qualifiée de mesure disciplinaire et elle doit de ce chef répondre aux prescriptions de l'article 10 LPJ. L'encadrement juridique de cette mesure est peu élaboré, puisque la seule exigence légale est que cette mesure doit être prise dans l'intérêt de l'enfant conformément à des règles internes affichées et expliquées à l'enfant. Aucune garantie procédurale, aucun principe de justice fondamentale, ne sont précisés. $D$ e manière générale, les règles internes sont brièvement rédigées autour de trois thèmes: respect de soi, des autres et de l'environnement (voir le code de vie des Centres jeunesse de Montréal à titre d'exemple). Un très grand nombre de comportements peuvent constituer un manque de respect de soi, des autres ou de l'environnement; en conséquence, la mesure de retrait peut être appliquée dans un nombre quasi illimité de situations. La mesure de retrait peut également servir des fins cliniques puisque dans plusieurs centres, le respect du code de vie est associé au développement du jugement moral et aux principes de la communauté juste. Le règlement sur la discipline des centres jeunesse de M ontréal spécifie qu'une mesure disciplinaire est une mesure éducative (CJM , 1994). Selon Jean-François B oulais, cette mesure n'aurait pas, dès lors, à être appliquée conformément à l'article 10 LPJ puisqu'elle n'est pas qualifiée de disciplinaire mais d'éducative (Boulais, 1999 : 94 et suivantes).

En termes pratiques, cela revient à dire que la mesure de retrait peut sanctionner n'importe quel comportement. Ajoutons à cela que la durée de la mesure de retrait n'est pas non plus encadrée par la loi. Lors d'une observation participante effectuée dans trois unités sécuritaires avec mon équipe de recherche (une d'arrêt d'agir, une d'encadrement intensif et une de garde fermée), j'ai noté des retraits de 1 heure, de 3 heures, de 15 heures. Contrairement à la mesure d'isolement, l'utilisation de la mesure de retrait ne fait l'objet d'aucune statistique annuelle. Bien que de ce fait son utilisation soit difficile à évaluer, j'ai pu constater qu'elle fait partie du quotidien dans certaines unités, général ement pour des périodes variant entre 1 et 3 heures, porte verrouillée, pour des motifs variés 
(manque d'efforts au sport, emprunt non autorisé d'une cigarette, appeler un autre jeune par son surnom).

En plus de ces mesures de retrait qui s'apparentent à l'isolement, il existe des programmes dits d' "arrêt d'agir». Les unités d'arrêt d'agir sont des unités à vocation temporaire (0-5 jours en principe). Les chambres des unités que nous avons visitées sont exemptes de tout contenu personnel (sauf dans les unités d'arrêt d'agir pour les 6-12 ans); on y trouve un lit de métal vissé au plancher, de même qu'un pupitre avec banc intégré, également vissé au plancher. Les portes des chambres se verrouillent automatiquement sitôt fermées, sans qu'on puisse les ouvrir de l'intérieur. Suivant la programmation d'une unité d'arrêt d'agir (Intervalle, centre D ominique-Savio-M ainbourg) et selon ce que nous avons pu y observer, les enfants sont enfermés dans leur chambre près de 20 heures par jour et doivent y rédiger des réflexions.

Cette période d'enfermement n'est pas considérée comme de l'isolement au sens de l'article 118.1 LSSSS. Elle n'est pas non plus toujours considérée comme une mesure disciplinaire. Selon le programme d'arrêt d'agir des Centres jeunesse de M ontréal, ce programme se définit comme une mesure spécifique de réadaptation qui «se vit dans des lieux physiques différents du milieu habituel du jeune» et qui «est utilisé en vertu de facteurs d'ordre clinique et il est intense, tant en matière de support que d'encadrement. Par son encadrement rigoureux, il vise l'interruption des actes déviants ou destructeurs par lesquels I'usager devient dangereux pour son entourage ou pour lui-même et contribue ainsi à détériorer sa situation. Par son support intensif, le programme permet à l'usager de faire le point sur son implication dans sa démarche et vise une concertation renouvelée de toutes les parties impliquées dans sa situation ». C'est, selon les termes employés, l' « intervention terminale» dans un «continuum de services» (CJM , 1998). Pourtant, dans certains Centres jeunesse dont celui de M ontréal, l'arrêt d'agir fait partie de la liste des mesures disciplinaires ${ }^{18}$.

Les mesures de retrait dans une pièce sécuritaire et verrouillée et l'arrêt d'agir sont toutes deux des mesures très privatives de liberté qui devraient être strictement encadrées de la même façon que la mesure

18. Centres jeunesse de M ontréal, Procédure d'application des mesures disciplinaires, art. $9.3 ;$ Centres jeunesse de l'Estrie, N ormes de pratique clinique sur les mesures disciplinaires à l'endroit des jeunes en centre de réadaptation, art. 9 ; Centres jeunesse de l'O utaouais, R ègles internes sur les mesures disciplinaires, art. 3. 
d'isolement. En effet, les conditions dans lesquelles certaines mesures de retrait et d'arrêt d'agir s'exercent s'apparentent à de l'isolement. Ce n'est pas la qualification de la mesure qui compte, mais son effet. Ainsi, malgré la diversité des vocables utilisés, la réalité demeure la même: I'enfant est isolé dans une pièce fermée à cl ef et vide de tout contenu personnel. Il ne faut pas perdre de vue que l'isolement n'est pas la salle d'isolement («un lieu») mais le fait d'être forcé à la solitude («un état»). II faut dépasser les frontières fictives de l'appellation de ces mesures pour les envisager pour ce qu'elles sont, des mesures d'isolement. Or, l'isolement disciplinaire est interdit par la loi. Le retrait est une mesure disciplinaire. Si le retrait s'effectue d'une manière qui équivaut à de l'isolement, alors cela devient de l'isolement disciplinaire déguisé.

En B elgique, il n'y a pas de programme s'apparentant à l'arrêt d'agir, ni de retraits dans des cellules nues. Le retrait en chambre est utilisé: le jeune doit alors faire une dissertation sur son comportement. L'outil privilégié pour assurer la discipline est la privation de sortie ${ }^{19}$. II existe dans les IPPJ un système très élaboré de cotation journalière de «bon comportement» selon une échelle allant de la note «T.B.» à «M al ». Ce système, dit-on, a une influence directe sur l'octroi des congés ${ }^{20}$. Selon le Règlement général du groupe des institutions publiques de protection de la jeunesse de la communautéfrançaise, «tout comportement positif ou résultat remarquable peut donner lieu à valorisation», alors que «tout comportement négatif peut faire l'objet de mesures disciplinaires». Dans les deux cas, les modalités de ces mesures sont fixées par chaque institution dans son règlement intérieur. À titre d'exemple, le Projet pédagogique du Service d'observation et d'orientation à régime fermé de I'IPPJ de Fraipont prévoit ce qui suit: «L'évaluation du comportement conduit, si nécessaire, à des sanctions sous forme de retrait de certains avantages, de l'imposition de tâches, de mise en chambre, de note au Juge. En contrepartie, lorsque le jeune fait montre de bonne volonté, il bénéficie d'un système de récompenses diversifiées», dont l'obtention de congés.

19. La fugue ou la tentative de fugue n'est pas sanctionnée. II faut dire qu'en droit belge l'évasion n'est pas un délit, encore moins la fugue.

20. Communauté française de Belgique, ministère de la Culture et des Affaires sociales, Institution publique de protection de la jeunesse de Fraipont, Projet pédagogique, art. 7.4. 


\section{Discussion}

De ce portrait sommaire, deux constats sautent aux yeux. Premièrement, le nombre de placements institutionnels de jeunes est beaucoup plus élevé ici qu'en Belgique et I'on recourt beaucoup plus à l'enfermement sécuritaire, quel que soit le nom que l'on donne à ces régimes: garde fermée, encadrement intensif, retrait, arrêt d'agir. D euxièmement, les ordonnances de placement en garde fermée sont de nature judiciaire en Bel gique et les mesures additionnelles de privation de liberté, telles l'isolement ou les transferts, sont solidement encadrées par la loi et font l'objet d'une décision de l'autorité de placement. Ici, à l'inverse, si l'isolement est encadré par la loi, toutes les autres mesures privatives de liberté ne sont l'objet d'aucune balise légale précise. $Y$ a-t-il un lien à faire entre ces deux constatations? II semble bien que oui.

$D$ ans leur traité sur le droit de la jeunesse, les auteurs Tulkens et M oreau (2001: 384) soulèvent la question suivante:

En entourant de certaines garanties les «mesures d'isolement dans des locaux spécifiques", c'est-à-dire, concrètement, la mise au cachot ou le placement en cellule nue, ces mesures sont implicitement admises et reconnues. $0 r$, fautil légaliser des pratiques honteuses ou persister dans le refus de leur acceptation? N ous pensons que de telles mesures, lorsqu'elles sont prévues dans les textes, auront un effet d'entraînement et qu'elles se multiplieront, selon la même logique qui veut que lorsqu'une prison s'ouvre elle se remplit aussitôt, quels que soient les besoins et quelles que soient les raisons.

$D$ 'après les conclusions d'une recherche subséquente (De Fraene et al., 2001), il semble que cette appréhension ne se soit pas confirmée jusqu'à maintenant, bien au contraire. Les chercheurs écrivent que « [l]'effet de ces textes sur les pratiques fut assez radical puisque, à partir de juillet 1989, les mesures d'isolement ont diminué de plus de $400 \%$, passant d'une moyenne mensuelle de 82 mesures pour l'ensemble des IPPJ à une moyenne de $19^{21} 》$. Cependant, ajoutent-ils, «[I]'utilisation d'autres pratiques substitutives s'est par contre accrue, dont la suppression de sorties extérieures et de week-end ainsi que les pratiques de mise à l'écart du groupe (la relance et la mise en chambre)».

Vu la situation au Q uébec, il me semble essentiel que I'utilisation des diverses formes d'isolement soit mieux balisée par la loi tant en ce qui

21. C'est aussi l'avis des directeurs que j'ai rencontrés, M. Demoulin, directeur de I'IPPJ Fraipont, et M. Joël Ponseele, directeur adjoint de l'IPPJ de Braine-le-Château. 
concerne leurs motifs, leur durée et l'aménagement architectural des lieux, qu'en ce qui concerne les modalités de prise de décision et le respect des principes de justice fondamentale. Le législateur doit reprendre en main son pouvoir normatif pour encadrer l'utilisation des mesures privatives de liberté et confier au seul pouvoir judiciaire le pouvoir d'enfermer un jeune dans un lieu sécuritaire.

Le droit québécois relatif aux mineurs privés de liberté est marqué par une faiblesse des fondements juridiques de ses normes: y abondent les règlements internes, les politiques ministérielles, les cadres de référence, les codes de vies, les pratiques et les usages. II y a quel ques années, le monde de l'enfermement pour adultes était ainsi caractérisé par la profusion, l'éparpillement et la faiblesse de ses normes. Le législateur canadien déléguait au commissaire des pénitenciers le pouvoir d'établir des règles concernant la garde, le traitement et la discipline des détenus. Les normes étaient inscrites dans des instructions, des ordres permanents, des directives souvent inaccessibles, qui n'avaient pas de statut «juridique» et n'étaient pas exécutoires (Lemonde, 1995 a; 1995 b).

Les détenus vivaient au royaume de l'arbitraire et de la délégation de pouvoir : des ordres et des directives, des pratiques et des règles, plutôt que des lois et des règlements, définissai ent le cadre de leur vie. Les décisions touchant directement les droits fondamentaux des prisonniers n'étaient pas prises par le Parlement, mais par une pléiade d'autorités déléguées qui opéraient largement hors des limites du contrôle du Parlement.

Le législateur canadien a procédé, en 1992, à une réforme majeure de la loi concernant l'incarcération et la libération conditionnelle et a ainsi repris son rôle de prédétermination des normes en ce domaine ( $L$ oi sur le système correctionnel, 1992). La nouvelle loi est très volumineuse et elle touche plusieurs sujets qui, auparavant, ne faisaient I'objet d'aucune norme ou ne se retrouvaient que dans les directives du commissaire. Ces dernières ne constituent plus le corpus normatif principal et, dans la plupart des cas, elles n'ajoutent plus que certains détails d'application de la loi. Contrairement à la situation ayant prévalu au cours des 150 années précédentes, les principales normes juridiques en vigueur dans les pénitenciers canadiens sont aujourd'hui d'ordre législatif plutôt qu'administratif.

Certains pays européens, comme la B elgique et la France, entre autres, sont en train de vivre cette transformation. Hergoz-Evans écrit que le droit pénitentiaire français est en pleine révolution (H ergoz-Evans, 1998; 2002). Contrairement aux autres branches du droit, le droit pénitentiaire 
restait marqué par une faiblesse des fondements juridiques des sources normatives et les sources réglementaires et les usages y dominaient. La France, écrit-elle, a fait le choix de ne pas organiser de débat démocratique sur des questions particulièrement sensibles, comme le régime coercitif appliqué aux détenus. Ce sont des préoccupations utilitaires et des considérations pratiques qui sont invoquées pour justifier ce choix: seules les «personnes terrain » peuvent donner des réponses adéquates aux problèmes pratiques et seul le réglementaire offre la souplesse adaptative nécessaire pour le maintien de l'ordre. Elle parle d'une «hiérarchie des normes total ement inversée: les usages locaux, voire les pratiques professionnelles individuelles primaient souvent sur les normes précises édictées par le Code de procédure pénale» (H ergoz-Evans, 2002).

Cette situation est celle que I'on retrouve aujourd'hui dans le monde de l'enfermement des jeunes au Q uébec. Chaque Centre jeunesse ${ }^{22}$ adopte ses propres règlements, inspirés d'une politique cadre de l'Association des Centres jeunesse, elle-même issue d'une lecture de rapports gouvernementaux (Commission Charbonneau, 1982; R apport Jasmin, 1992). Et, à l'intérieur de chacun des 18 Centres jeunesse, chaque unité des divers centres de réadaptation élabore, suivant I'orientation du C entre, ses propres règles, son propre «code de vie», son propre niveau d'encadrement, son propre programme.

La «légal isation » des droits des mineurs privés de liberté et la «judiciarisation » du placement sécuritaire ne constituent évidemment pas une solution miracle. En Angleterre, le placement et le maintien des jeunes en milieu sécuritaire doit être le fruit d'une décision judiciaire. Une équipe a mené une recherche sur le sujet et a observé les audiences judiciaires. Leurs conclusions sont assez sévères: le raffinement de la législation n'a pas d'effet significatif sur les pratiques et les juges ne remettent pas en question l'opinion des professionnels et des travailleurs sociaux. Pour les chercheurs, il vaudrait mieux se concentrer sur la réduction de la population en milieu sécuritaire que sur les garanties procédural es entourant leur admission en ces lieux (H arris et Timms, 1993).

II semble donc que la judiciarisation du processus soit impuissante à elle seule à assurer la protection du droit à la liberté. D es balises juridiques plus contraignantes entourant l'admission et l'utilisation de mesures sécu-

22. Le territoire du $Q$ uébec est divisé en 18 régions dites «socio-sanitaires» qui ont chacune un $C$ entre jeunesse. Au sein de chaque Centre jeunesse, I'on retrouve un ou plusieurs Centres de réadaptation selon la densité de la population. Ainsi, les centres jeunesse de M ontréal comptent quatre centres de réadaptation qui comprennent à leur tour plusieurs unités de vie. 
ritaires, comme c'est le cas en Belgique, ont cependant deux effets certains: le taux de placement des jeunes en institution est réduit de façon importante et le recours aux mesures additionnelles de privation de liberté est moins fréquent.

Lors d'une institutionnalisation, les mesures additionnelles de privation de liberté ne devraient pas constituer la seule alternative pour répondre aux besoins des enfants et des adolescents, principalement ceux qui ont besoin de protection, mais elles continuent à être un mode habituel quasi inévitable de régulation dans les établissements pour mineurs. Un encadrement juridique plus serré et plus conforme à la réal ité pourrait permettre de réduire l'utilisation de ces mesures.

II faut, de plus, viser la réduction de l'enfermement des jeunes et, comme nous le montre l'exemple belge, seule une véritable volonté politique et sociale, reflétée dans une loi stricte et accompagnée d'un investissement sérieux dans les ressources externes et d'un transfert des expertises, pourra permettre d'y arriver. L'exemple belge nous montre aussi que la vigilance constante de divers acteurs sociaux, groupes de défense des droits des jeunes, militants, intellectuels, avocats et autres, couplée d'un dialogue avec les intervenants politiques et professionnels sont essentiels pour contrecarrer la pression publique réclamant une plus grande répression à l'endroit des mineurs.

\section{Références}

A ccord de coopération entre l'État fédéral, la Communauté germanophone, la Communauté française et la Communauté flamande relatif au centre ferme pour le placement provisoire de mineurs ayant commis un fait qualifié d'infraction.

A rrêté du $G$ ouvernement de la Communauté françai se réglementant les modal ités d'isole ment dans les I nstitutions publiques de protection de la jeunesse, organisant le contrôle de ces modalités et fix ant les normes applicables aux salles d'isolement, 21 mars 1997.

Association des centres jeunesse du Q uébec (1995). Cadre de référence pour une politique et procédure relative à l'isol ement des usagers. $Q$ uébec.

Bandeira, M ., \& O uellet, R. (1986). Analyse systématique de la procédure d'isolement et de son application en établissement. Revuede modification du comportement, 16, 5.

B oulais, J.-F. (1999). Loi de protection de la jeunesse, tex te annoté, 4 e édition. M ontréal : SO QIJ.

Centre de recherche sur les droits et libertés (1985). G randir à l'ombre de la famille et del'É tat. Q uébec: Université Laval. 
Centres jeunesse de M ontréal (1994). Règlement no 4 portant sur les mesures disciplinaires.

Centres jeunesse de M ontréal (1995). Règl ement no 7 relatif à l'utilisation de l'isole ment d'un usager des centres jeunesse de M ontréal.

Centres jeunesse de M ontréal (1998). Programme d'arrêt d'agir.

Communauté française de Belgique, ministère de la Culture et des Affaires sociales, Institution publique de protection de la jeunesse de F raipont, Projet pédagogique.

Crespi, T.D. (1988). Effectiveness of time-out: a comparison of psychiatric, correctional and day-treatment programs. A dolescence, 23, 805.

Décret du 4 mars 1991 de la Communauté française relatif à l'aide à la jeunesse.

De Fraene, D., De M an, C., \& Thibaut, C. (2001). Pratiques dela sanction dans les institutions publiques de protection de la jeunesse. $C$ entre de recherches criminologiques de l'U niversité Libre de Bruxelles.

Exposé des motifs, 1990-1991. D oc. Cons. Comm. Fr., 165, n¹.

Graindorge, A. (2001). Réforme de la loi du 8 avril 1965. Dépasser le débat protection/ sanction - un pas difficile. Journal du D roit des Jeunes, 203, 3-19.

Harris, R., \& Timms, N. (1993). Secure accomodation in child care: between hospital and prison or thereabouts? London: Routledge.

H erzog-Evans, M. (1998). Les particularités du droit pénitentiaire. Les cahiers de la securitéintérieure, Prisons en société, 31, 19-34.

H erzog-Evans, M . (2002). La révolution pénitentiaire française. In 0. De Schutter \& D. Kaminski (eds), L'institution du droit pénitentiaire. Enjeux de la reconnaissance de droits aux détenus (17-41). Paris: Bruylant, L.D.G.D.

Kennedy, P., Kupst, M.J., Westman, G., Zaar, C., Pines, R., \& Schullman, J.L. (1990). U se of the time out procedure in a child psychiatry inpatient milieu: combining dynamic and behavioral approaches. Child Psychiatry and $\mathrm{H}$ uman D evelopment, 20, 207.

Lemonde, L. (1995a). L'évolution des normes dans l'institution carcérale. Revue canadienne D roit et Société / Canadian Journal of Law and Society, 10 (1), 125170.

Lemonde, L. (1995b). H istorique des normes juridiques dans les pénitenciers au Canada. Criminologie, xxvIII (1), 97-117.

Lemonde, L. (2001). Le droit à un recours effectif lors de la violation des droits fondamentaux. M ini-colloque. Ledroit dela jeunesse. Barreau du Q uébec, Service de la formation permanente, avril 2001.

Loi sur les jeunes contrevenants, L.R.C. (1985), C. Y-1.

Loi sur les services de santé et les services sociaux, L.R.Q., C. S-42.

Loi sur la protection de la jeunesse, L.R.Q., C. P-34.1.

Loi du 8 avril 1965 relativeà la protection de la jeunesse.

Loi du 8 août 1980 des réformes institutionnelles.

Loi du 2 février 1994.

Loi sur le système correctionnel et la mise en liberté sous condition, S.C., (1992), C. 20.

Loi sur le système de justice pénal e pour adolescent, C-7, 2002. 
M ace, F.C., \& Heller, M . (1990). A comparison of exclusion time-out and contingent observation for reducing severe disruptive behavior in a 7 year-old boy. Child \& Family Behavior Therapy, 12, 57.

M. V. C. Diane G agnon, D.P.J., CA 500-08-000138-000.

M essier, C., \& Toupin, J. (1994). La clientèle multiethnique des centres de réadaptation pour les jeunes en difficulté. Q uébec: Commission de protection de la jeunesse.

Protection de la jeunesse- 193 [1986] R.J.Q. 736 (C.S.).

Q uébec (1982). Rapport de la Commission parlementaire spéciale sur la protection de la jeunesse: aspects historiques (Commission Charbonneau). Q uébec: Assemblée nationale.

Q uébec (1992). Rapport du groupe de travail sur l'évaluation de la Loi de protection de la jeunesse, La protection de la jeunesse, Plus qu'une loi (R apport Jasmin). Q uébec: M inistère de la Santé et des Services sociaux et ministère de la Justice.

Trépanier, J., \& Tulkens, F. (1995). Délinquance et protection de la jeunesse. A ux sources des lois belge et canadienne sur l'enfance. Bruxelles: D eB oeck U niversité.

Tulkens, F., \& M oreau, T. (2001). D roit de la jeunesse. Bruxelles: Larcier. 